\title{
Evaluation of accessible regions of Escherichia coli fimH mRNA through computational prediction and experimental investigation
}

\author{
Elnaz Harifi Mood, Alireza Japoni-Nejad, Mohammadreza Asadi Karam, Mohammad Pooya, Saeid \\ Bouzari*, Nader Shahrokhi*
}

Department of Molecular Biology, Pasteur Institute of Iran, Tehran, Iran

Received: May 2021, Accepted: August 2021

\begin{abstract}
Background and Objectives: This study aimed to investigate the accessible regions of the fimH mRNA using computational prediction and dot-blot hybridization to increase the effectiveness of antisense anti-virulence therapeutics against Uropathogenic Escherichia coli.

Materials and Methods: We predicted the secondary structure of the E. coli fimH mRNA using the Sfold and Mfold Web servers and RNA structure 5.5 program. Considering the predicted secondary structure, accessible regions in mRNA of fimH were determined and oligonucleotides complementary to these regions were synthesized and hybridization activity of those oligonucleotides to the fimH Digoxigenin (DIG) labeled mRNA was assessed with dot-blot hybridization.

Results: When searching the fimH gene in the GenBank database, two lengths for this gene was discovered in different strains of E. coli. The difference was related to the nine bases in the first part of the gene utilizing either of two translation initiation sites. Based on the bioinformatics analyses, five regions lacking obvious stable secondary structures were selected in mRNA of $f i m H$. The result of dot-blot hybridization exhibited strongest hybridization signal between the antisense oligonucleotide number one and fimH labeled mRNA, whereas hybridization signals were not seen for the negative control.

Conclusion: The results obtained here demonstrate that the region contains start codon of fimH mRNA could act as the potential mRNA target site for anti-fimH antisense therapeutics. It is recommended in the future both of utilizing translation initiation sites be targeted with antisense oligomers compounds.
\end{abstract}

Keywords: Uropathogenic Escherichia coli; FimH protein; Target prediction; Nucleic acid hybridizations

\section{INTRODUCTION}

Urinary tract infections (UTIs) including cystitis (bladder infections) and pyelonephritis (kidney infections) are the most common bacterial infections which affect 150 million people each year worldwide $(1,2)$. More than $50 \%$ of women and $15 \%$ of men will experience at least one UTI during their lifetime (3). Moreover, nearly one-half of those women will go on to develop one or more additional UTIs at a later time underlining the high rate of recurrent UTI (Recurrent UTI is defined by the occurrence of more than two episodes in 6 months, or three in 12-months) $(4,5)$. UTI accounts for nearly $40 \%$ of all hospitals acquired infections and $50 \%$ of bacteremia that can extend the hospitalization and rise the morbidity and mortality rate of patients (6).

UTIs can be originated by a diverse species of bac-

*Corresponding author: Saeid Bouzari, Ph.D, Department of Molecular Biology, Pasteur Institute of Iran, Tehran, Iran. Tel: +98-2164112220Ｆax: +98-2164112803_Email: bouzari@pasteur.ac.ir

"Corresponding author: Nader Shahrokhi, Ph.D, Department of Molecular Biology, Pasteur Institute of Iran, Tehran, Iran. Tel: +98-9123847794Ｆax: +98-2164112803Ｅmail: shahrokhi@pasteur.ac.ir 
teria, but Uropathogenic Escherichia coli (UPEC) is the leading etiologic agent of these infections (more than $85 \%$ of community-acquired UTI and $50 \%$ of nosocomial UTI), while other Gram-negative bacteria (e.g., Proteus mirabilis, Klebsiella pneumoniae) and Gram-positive cocci (e.g., Staphylococcus saprophyticus, Enterococcus faecalis) are accountable for the remaining cases (7-9).

UPEC strains have a capability to encode wide range of virulence factors that connected to colonization, persistence, and pathogenesis of bacteria in the urinary tract $(10,11)$. Type 1 pili, are owned by a family of bacterial adhesive organelles named chaperone-usher pili, encoded by the fim operon, which play critical role for effectual colonization and pursuant disease progress in mucosal infections (12).

The FimH protein, the receptor-recognizing element of type 1 fimbriae, is placed at the very distal tip of type 1 pilus. This adhesion exhibits robust affinity for mannosylated glycoproteins on the surface of human bladder cells, easing bacterial colonization, invasion, and formation of biofilm-like intracellular bacterial communities (IBCs) $(8,11)$. IBCs are protected from infiltrating phagocytes and are possibly less sensitive to antibiotic treatments (1). These pili also permit the bacteria to attach onto inanimate surfaces such as catheters (11).

High rate of recurrence is an especially disturbing feature of UPEC-associated UTIs. This problem is exacerbated via the drastic rise in the worldwide incidence of antibiotic-resistant UPEC strains over the past two decades (1). Consequently, there is an urgent need to develop novel therapeutic strategies for treating and managing UPEC infections and most particularly recurrent UTIs.

RNA-based antibiotics in the form of short antisense oligonucleotides (ASOs) are an interesting technology that inhibit target genes on the RNA level (13). In this approach, single-stranded antisense oligonucleotides are often chemically modified to gain stability in biological media (14). Binding ASOs to the complementary target mRNA through base pairing mechanism can cause of specific inhibition of gene expression by different mechanisms, depending on the chemical structure of the ASO, resulting in reduced levels of translation of the target transcript. Numerous mRNA encoding essential and non-essential/virulence genes in clinically pathogenic bacteria have been corroborated as promising targets for antisense antibacterial agents in the last decades (15-21).
The most effective antisense agents were designed as complementary to mRNA around and proximally upstream of the translation start codon (22). Recently, some researcher pointed out that the full length of a target gene screening using computational prediction and dot-blot hybridization can help us to select potent and specific ASOs compounds (19, 23-25).

Considering the critical role of FimH adhesion for initiating and sustaining UPEC infections, this virulence factor could be an attractive target for the development of anti-virulence antisense therapeutics. Therefore, in this study, we have screened the accessible regions of the fimH mRNA of E. coli UTI89 strain with the ultimate aim of increasing effectiveness of anti-fim $H$ antisense therapeutics in future.

\section{MATERIALS AND METHODS}

Computational screening of accessible regions in the fimH mRNA. Multiple sequence alignment of fimH gene, obtained from various $E$. coli strains, was carried out using the Basic Local Alignment Search Tool (BLAST) on the NCBI website (26) to identify the conserved regions. The secondary structure of the E. coli fimH mRNA was predicted using the Mfold Web server (http://www.unafold.org) and RNA structure 5.5 programs based on minimal free energy approaches $(27,28)$ and the Sfold Server (http://sfold.wadsworth.org) based on structure sampling algorithms (29). The Sfold Server provides tools for the rational design of antisense oligos by combining prediction of target secondary structure and accessibility with empirical design rules. RNA structure is a comprehensive package for RNA secondary structure prediction and free energy analysis. It contains algorithms for prediction of secondary structure, comprising facility to predict base pairing probabilities (30). The targets requirements contain overall $\Delta \mathrm{G} \leq-10 \mathrm{kcal} / \mathrm{mol}$, oligo-self $\Delta \mathrm{G} \geq-2 \mathrm{kcal} /$ mol, oligo-oligo $\Delta \mathrm{G} \geq-10 \mathrm{kcal} / \mathrm{mol}, \mathrm{GC} \%=40-60 \%$ and $\mathrm{Tm}>50^{\circ} \mathrm{C}$. According to the predicted secondary structure and binding energy $(\Delta \mathrm{G} 37)$ prediction, the regions lacking obvious stable secondary structures in mRNA of fimH were selected (they were theoretically accessible to complementary antisense). Moreover, one oligonucleotide from the sense strand sequence was used as a negative control. These antisense oligonucleotides which targeted the predicted accessible regions of fim $\mathrm{H}$ mRNA and one oligonu- 
cleotide as a negative control were synthesized and assessed by dot-blot hybridization for their binding activities to fimH mRNA.

fimH mRNA DIG labeling via in vitro T7 transcription. E. coli UTI89 strain was grown in $5 \mathrm{ml}$ of Luria Bertani (LB) broth medium. The genomic DNA of E. coli UTI89 was extracted with bacteria DNA Kit (Invitrogen), according to the manufacturer's protocol. The full length fim $H$ gene was amplified by touchdown PCR with the forward primer containing the $\mathrm{T} 7$ promoter fused to a specific sequence complementary to the sequence of fimH gene (Table 1). The primers were designed based on the fimH gene of UTI89 strain (GenBank accession number NC_007946.1). PCR amplification was carried out in $50 \mu \mathrm{l}$ volume containing $2 \mu \mathrm{l}$ of DNA template, $5 \mu \mathrm{l}$ of $10 \times$ reaction buffer, $2 \mu \mathrm{l}$ of dNTPs $(10 \mathrm{mM}), 2$ $\mu \mathrm{l}$ of $\mathrm{MgCl}_{2}(50 \mathrm{mM}), 2 \mu \mathrm{l}$ of each primer (10 pmol), and $1 \mathrm{U}$ of $p f u$ DNA polymerase (Fermentas). After amplification of fimH gene, $5 \mu \mathrm{l}$ of sample was subjected to electrophoresis on a $1 \%$ agarose gel to confirm the presence of the amplified product.

The purification of the PCR product was performed with high pure PCR product purification kit (Roche) and after sequencing was used as a template for in vitro transcription. The fimH mRNA was transcribed in vitro with DIG RNA labeling kit (Roche) in the presence of digoxigenin-UTP (DIG-UTP), according to the manufacturer's instructions (31). Briefly, a $20 \mu \mathrm{l}$ reaction was set up containing $2 \mu \mathrm{l}$ of $10 \times$ transcription buffer, $2 \mu \mathrm{l}$ of $10 \times$ NTP labeling mix- ture, $1 \mu \mathrm{l}$ of protector RNase inhibitor, $1 \mu \mathrm{g}$ of purified template DNA, and $2 \mu 1$ of T7 RNA polymerase (The reaction vial was placed on ice). Nuclease-free water was added to a final volume of $20 \mu \mathrm{l}$. After incubation at $37^{\circ} \mathrm{C}$ for $2 \mathrm{~h}$, the template DNA was removed by digestion with DNase I for $20 \mathrm{~min}$ at $37^{\circ} \mathrm{C}$ and then reaction was stopped by adding $2 \mu 10.2 \mathrm{M}$ EDTA ( $\mathrm{pH}$ 8) (24). The RNA transcripts were quantified, and stored as aliquots in nuclease-free water at $-80^{\circ} \mathrm{C}$ until analysis. Schematic overview of the fim $\mathrm{H}$ mRNA DIG labeling and in vitro $\mathrm{T} 7$ transcription is shown in Fig 1. The instruction manual for DIG RNA labeling kit is provided by the manufacturer and available at www.roche-applied-science.com. Homology of fim $H$ gene coding region among $E$. coli strains is shown in Table 2.

Dot-blot hybridization. Dot-blot hybridization was performed using the DIG system (Roche) (31). All reagents except buffers were included in the kit. In this study, the DIG labeled fimH mRNA was obtained in the in vitro $\mathrm{T} 7$ transcription, used as a probe in dot-blot hybridization with the antisense oligonucleotides as a targets. The antisense oligonucleotides and negative control listed in Table 3. Two $\mu \mathrm{g}$ of antisense oligonucleotides and also one oligonucleotide as a negative control (oligo number 6) were spotted on positively charged nylon membrane (Roche). Afterwards, the membrane was placed on a Whatman paper soaked with $10 \times$ sodium citrate/sodium chloride (SSC), and the denatured DNA was fixed to the membrane by UV cross-linking for 3-5 min. After

Table 1. (A) The primers used for amplification of fim $H$ gene for doing in vitro transcription. The full length fimH gene was amplified by touchdown PCR with the forward primer containing the T7 promoter fused to a specific sequence complementary to the sequence of $f i m H$ gene.

\begin{tabular}{lcc}
\hline Primer/sequence & PCR product & Reference \\
\hline fimH -F: ATGAAACGAGTTATTACCCTGTT & $903 \mathrm{bp}$ & This study \\
fimH -R: TTATTGATAAACAAAAGTCACGCC & & \\
T7promotor +fimH-F: & $933 \mathrm{bp}$ & This study \\
TAATACGACTCACTATAGGGCTAGAAGGAGATGAAACGAGTTATTACCCTGTT & & \\
fim $H$-R: TTATTGATAAACAAAAGTCACGCC & & \\
\hline
\end{tabular}

(B): The touchdown PCR program for doing in vitro transcription

\begin{tabular}{lccc}
\hline Cycle number & Denaturation & Annealing & Polymerization \\
\hline 1 & 6 min at $95^{\circ} \mathrm{C}$ & $30 \mathrm{~s}$ at $62^{\circ} \mathrm{C}$ & $55 \mathrm{~s}$ at $72^{\circ} \mathrm{C}$ \\
$2-34$ & $30 \mathrm{~s}$ at $95^{\circ} \mathrm{C}$ & $35 \mathrm{~s}$ at $62^{\circ} \mathrm{C}\left(\right.$ decrease temperature $0.5^{\circ} \mathrm{C} /$ cycle $)$ & $55 \mathrm{~s}$ at $72^{\circ} \mathrm{C}$ \\
35 & $30 \mathrm{~s}$ at $95^{\circ} \mathrm{C}$ & $35 \mathrm{~s}$ at $45^{\circ} \mathrm{C}$ & 8 min at $72^{\circ} \mathrm{C}$ \\
\hline
\end{tabular}


cross-linking, any unbound oligonucleotides were removed by two times washing in $0.5 \times \mathrm{SSC}, 0.1 \%$ sodium dodecyl sulfate (SDS) for $2 \mathrm{~min}$ at $37^{\circ} \mathrm{C}$. After pre-hybridization at $37^{\circ} \mathrm{C}$ for $30 \mathrm{~min}$ in DIG Easy Hyb solution (Roche) with gentle agitation, the membrane was hybridized with $100 \mathrm{ng} / \mathrm{mL}$ of DIG-UTP labeled

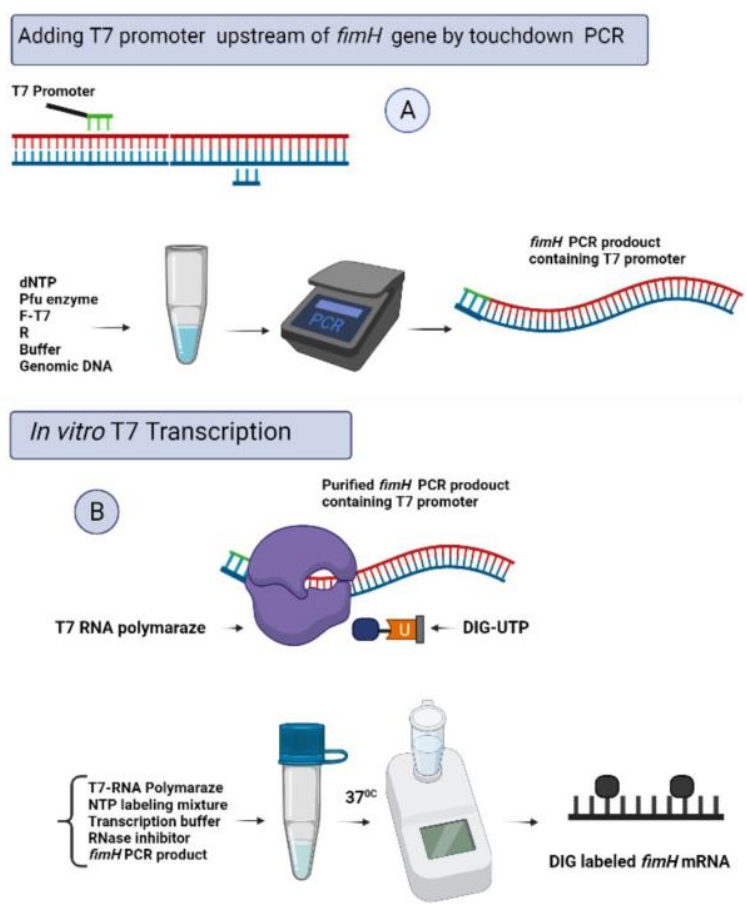

Fig. 1. Schematic overview of the herein applied strategy. (A) Amplification of the whole length of fim $H$ gene by PCR using forward primer containing the $\mathrm{T} 7$ promoter sequence. The purified PCR product was used as a template in the next step. (B) fimH mRNA DIG labeling and in vitro T7 transcription was performed by using DIG RNA labeling kit (Roche). The main parts of the image have been created with BioRender.com.
fimH mRNA in DIG Easy Hyb solution at $37^{\circ} \mathrm{C}$ for $6 \mathrm{~h}$, washed twice in $2 \times \mathrm{SSC}$ and $0.1 \% \mathrm{SDS}$ at room temperature for $10 \mathrm{~min}$ each, and then twice in $0.5 \times$ SSC and $0.1 \%$ SDS at $65^{\circ} \mathrm{C}$ for $20 \mathrm{~min}$ each. Then hybridization signal was identified by using the DIG nucleic acid colorimetric Detection kit (Roche) (32). The membrane was incubated $30 \mathrm{~min}$ in the blocking solution. Approximately $0.5 \mathrm{ml}$ of alkaline phosphatase conjugated sheep anti-DIG antibodies (diluted 1:5000 in blocking solution) was added and the membrane was incubated at $37^{\circ} \mathrm{C}$ for $30 \mathrm{~min}$. After $2 \times 15$ min washing, equilibrated $5 \mathrm{~min}$ in detection buffer and then incubated in freshly prepared color substrate solution (containing NBT/BCIP) in appropriate container in the dark (Color development) was clearly visible between $30 \mathrm{~min}$ and $1 \mathrm{~h}$ after the start of the reaction).

\section{RESULTS}

Computational screening of accessible regions in the fimH mRNA. The fimH gene is well conserved across $E$. coli strains. The BLASTN results confirmed the $5^{\prime}$ terminal region of the fim $H$ gene including the ribosomal binding site and the start ATG codon is highly conserved among $E$. coli strains, which suggested that fimH would be a specific antisense target. Nevertheless, the bioinformatics analysis revealed the considerable gene size characteristics of fimH gene in $E$. coli strains. When we searched fimH gene in Gene database in NCBI, we realized this gene has two lengths in different strains of $E$. coli (Table 2). The fimH gene in E. coli CFT073 (UPEC) and E. coli 536 (UPEC) strains was 912 bp in length while that was 903 bp in length on the other strains (Fig. 2). The dif-

Table 2. Homology of fimH gene coding region among E. coli strains

\begin{tabular}{lccc}
\hline Strains & Genbank & Gene length $(\mathbf{b p})$ & Identity $(\%)$ \\
\hline E. coli ( K-12,MG1655) & NC_000913.3 & 903 & 99 \\
E. coli CFT073 & AE014075.1 & 912 & 99 \\
E. coli UTI89 & NC_007946.1 & 903 & 99 \\
E. coli 536 (UPEC) & CP000247.1 & 912 & 99 \\
E. coli LF82 & CU651637.1 & 903 & 99 \\
E. coli strain S88 & CU928161.2 & 903 & 99 \\
E. coli W3110 & NC_007779.1 & 903 & 99 \\
E. coli O139:H28 E24377A & JXRF01000007.1 & 903 & 99 \\
E. coli O7:H4 strain MIN14 & CP069646.1 & 903 & 99 \\
E. coli ATCC 8739 & CP043852.1 & 903 & \\
\hline
\end{tabular}


(A)

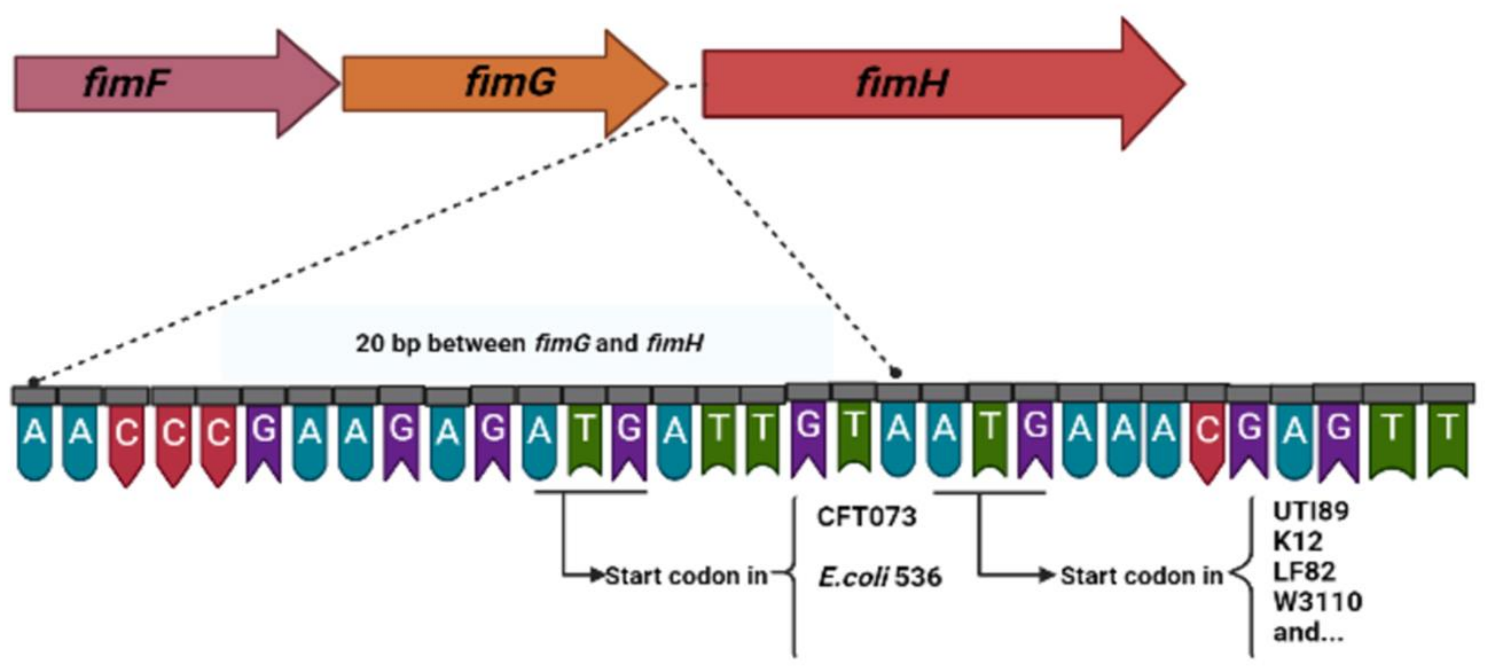

(B)
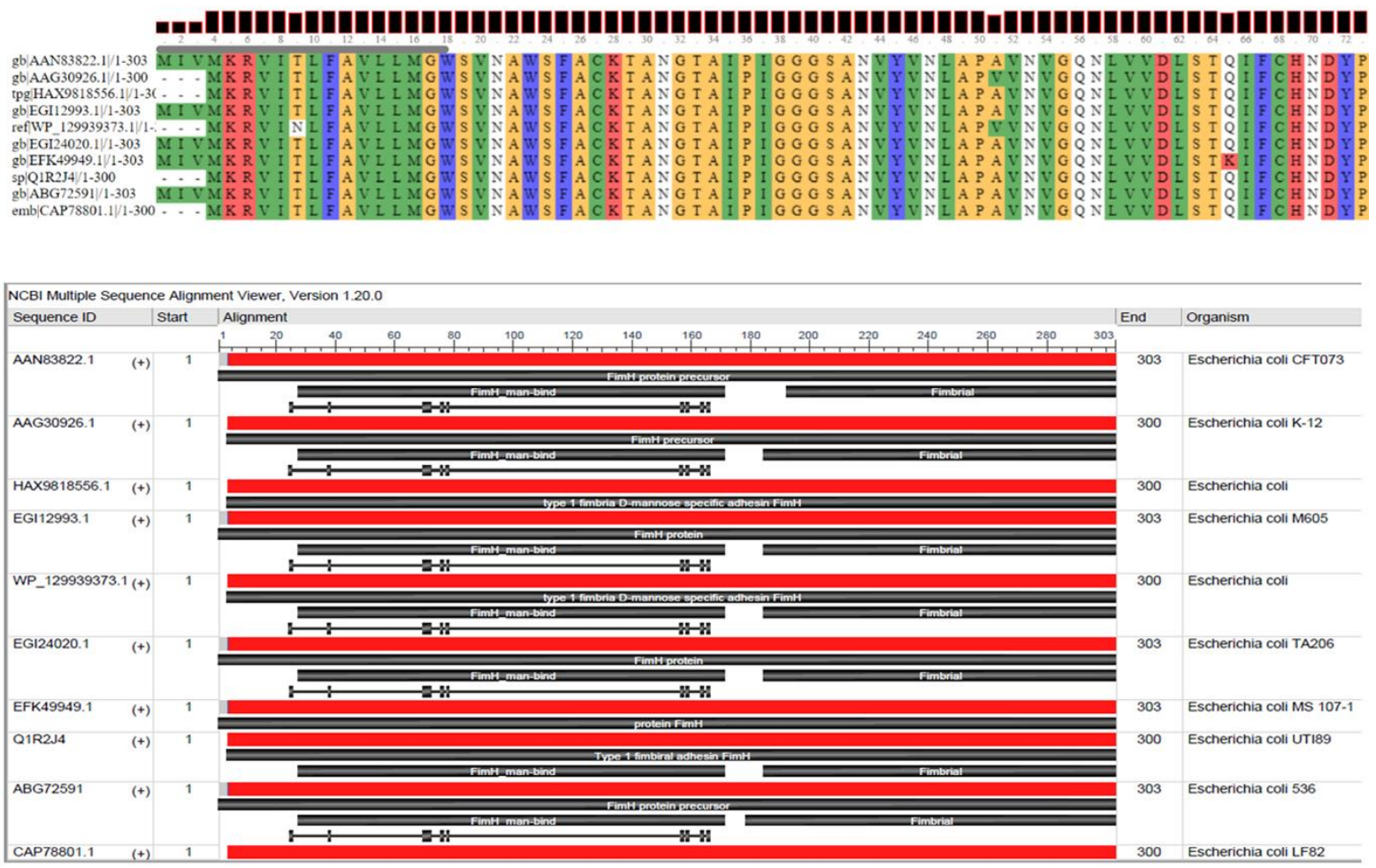

Fig. 2. (A) Schematic of the fimH gene in E. coli UTI89 strain. The oligonucleotide sequence (20 bp) exist between fimG and fim $H$ genes is shown here. When searching the fimH gene in the NCBI (GenBank) database, two lengths for this gene in different strains of $E$. coli were discovered. The fimH coding gene in most of $E$. coli strains such as K12, UTI89, W3110 and LF82 was 903 bp in length, while the length was 912 bp for both E. coli CFT073 (UPEC) and E. coli 536 (UPEC) strains. The difference was related to the nine bases in the first part of the gene utilizing either of two translation initiation sites. (B) Multiple amino acid sequence alignment of FimH protein by COBALT server. As shown in the figure, FimH protein contains 300 amino acids in E. coli K12 (UniProt ID: AAG30926.1) and UTI89 (UniProt ID: Q1R2J4) strains, while FimH protein consists of 303 amino acids in E. coli CFT073 (UniProt ID: AAN83822.1) and E. coli 536 (UniProt ID: ABG72591) strains. 
ference was related to the nine bases in the first part of the gene utilizing either of two translation initiation sites (Fig. 2).

We predicted the secondary structure of the E. coli UTI89 fimH mRNA using the Sfold softwear (Fig. 3A), RNA structure programs (Fig. 3B) and Mfold software (Fig. 4). Successful ASO design needs to consider thermodynamic energy. So, the program OligoWalk from the package RNA structure was used to calculate thermodynamic properties between the mRNA target sequence and ASO. To design a potent ASO, the binding energy between the ASO and mRNA should be $\Delta \mathrm{G} 37 \geq-8 \mathrm{kcal} / \mathrm{mol}$, whereas the energy for binding between ASOs should be $\Delta \mathrm{G} 37 \geq-1.1$ $\mathrm{kcal} / \mathrm{mol}$ (Fig. 3F). According to the predicted secondary structure and thermodynamic properties, five regions lacking obvious stable secondary structures in mRNA of fimH were selected (predicted regions included bulges, internal and external loops and joint sequences-Fig. 4). Antisense oligonucleotides which targeted the five regions of fim $\mathrm{H}$ mRNA and one oligonucleotide as a negative control were represented in Table 3. These six oligonucleotides were synthesized and their binding activities to fimH labeled mRNA evaluated by dot-blot hybridization.

fimH mRNA DIG labeling via in vitro T7 transcription and dot-blot hybridization. In this study, we used PCR based in vitro transcription. The forward primer was linked to a $\mathrm{T} 7$ promoter sequence (5'-TAATACGACTCACTATAGGG-3') for incorporating a T7 promoter into a PCR product of fimH (Fig. 5). The principle of dot-blot hybridization in this study was that the DIG labeled mRNA of fimH can bind to the DNA oligonucleotides (Table 3) that contain a complementary sequence, and the hybrid stability depending on the magnitude of binding that occurs. Experimentally, the analysis was performed with a labeled probe and target DNAs that immobilized on a membrane support.

First, we estimated the yield of DIG labeled fimH mRNA compared to the labeled RNA control (provided with the Roche kit) by dot-blot hybridization and comparison of the spot intensities. We concluded that our DIG labeled fimH mRNA yield was approximately $100 \mathrm{ng} / \mu \mathrm{L}$ (yield estimation is the mean of two individual experiments).

In the next step, we carried out Dot-blot hybridization to investigate the binding activity of predicted antisense oligonucleotides and negative control (Ta- ble 3) with $E$. coli UTI89 fimH DIG labeled mRNA. Two $\mu$ g of each denatured antisense oligonucleotides (number 1 to 5) and oligonucleotide number 6 (as negative control) were spotted and fixed to the membrane (Fig. 6) then the membrane was hybridized with $100 \mathrm{ng} / \mathrm{mL}$ of DIG labeled fimH mRNA in DIG Easy Hyb solution(Roche). As shown in Fig. 6, five oligonucleotides exhibited different degree of hybridization signals on the dot-blot hybridization. Among them, the oligonucleotide number 1 (target size 1-18) that targeted the region contains start codon of fim $\mathrm{H}$ mRNA showed the strongest hybridization signal, whereas the negative control (with a sense sequence) showed no hybridized signal. The start codon region of fimH mRNA was also predicted by the RNA structure program as a site with highest probability to be accessible with antisense oligonucleotide (Fig. 3F). Considering that we concluded the region contains start codon of fimH mRNA as the ideal mRNA target site for antisense suppression compares to the other sites.

\section{DISCUSSION}

There are few effective treatment choices for treating the chronic and recurrent urinary tract infections caused by UPEC strains. Patients with chronic urinary tract infections are commonly treated with long-term prophylactic antibiotics that promote the development of antibiotic-resistant forms of UPEC, further complicating treatment (33). Furthermore, a history of UTI significantly predisposes one to recurrent UTI. Thus, antibiotic sparing approaches, such as anti-virulence strategies that prevent UPEC colonization, represent viable therapeutics to address this emerging threat (34). Herein, we determined secondary structure motifs and accessible regions of the $E$. coli fim $H$ mRNA with the ultimate aim of increasing effectiveness of anti-fim $H$ antisense therapeutics in future.

The research on anti-virulence strategies in UTIs has focused on different steps of bacterial pathogenesis such as colonization, adherence of pathogens to cell receptors and invasion (5). Targeting bacterial virulence is an alternative approach to antimicrobial therapy that offers promising opportunities to inhibit pathogenesis and its consequences without placing immediate life or death pressure on the target bacterium $(11,35)$. 

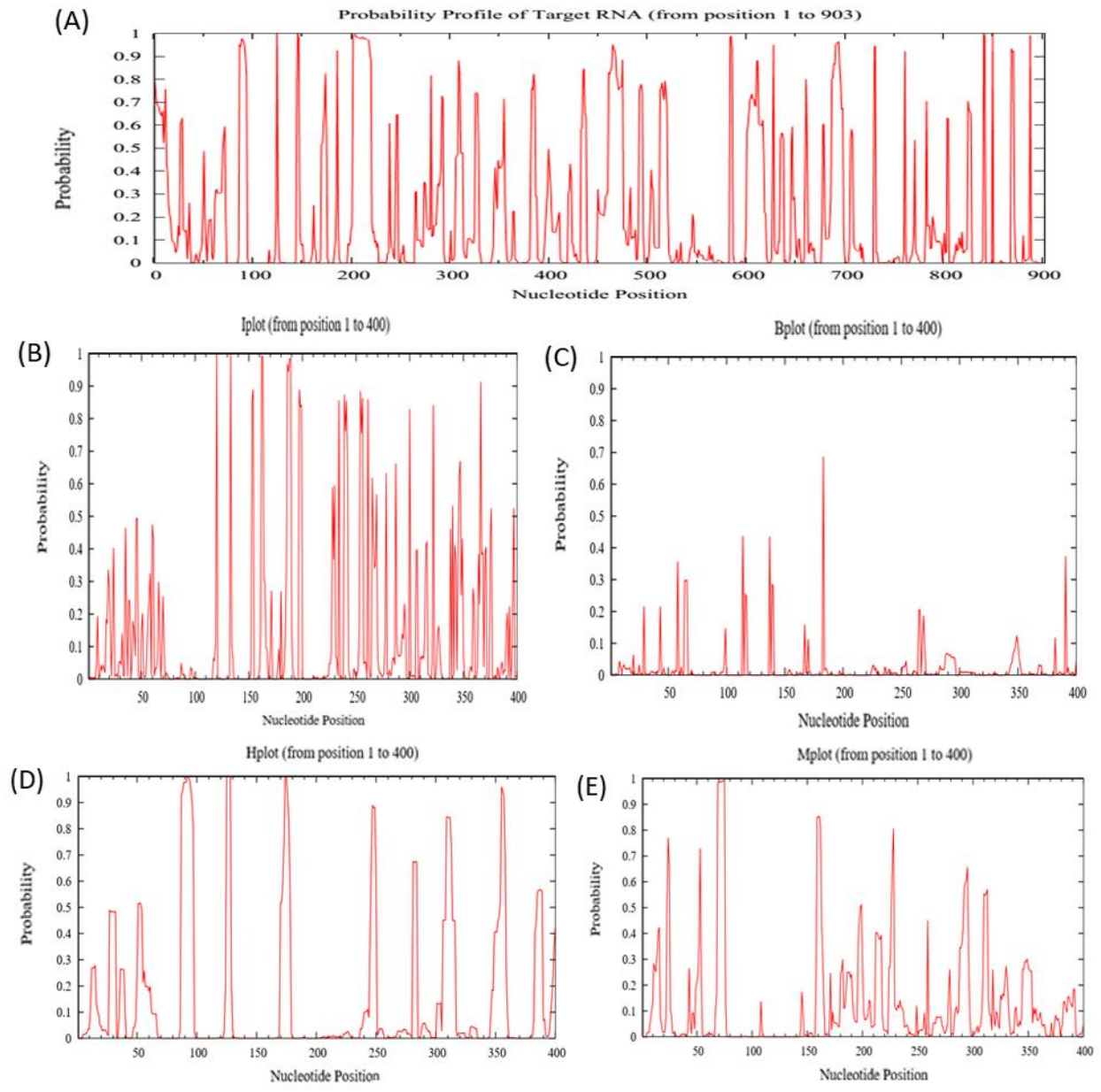

(F)

\begin{tabular}{ccc}
\hline Position on target & Probability of being efficient Antisense & Antisense Sequence(5'->3') \\
340 & $5.15539 \mathrm{e}-05$ & UUAUAAACAAC \\
3 & $2.8302 \mathrm{e}-05$ & UAACUCGUUUC \\
339 & $2.5038 \mathrm{e}-05$ & UAUAAACAACC \\
354 & $2.16914 \mathrm{e}-05$ & UAUCCGUUCUC \\
684 & $1.69233 \mathrm{e}-05$ & UGGUGAAAAUC \\
681 & $1.5489 \mathrm{e}-05$ & UGAAAAUCGAG \\
338 & $1.23958 \mathrm{e}-05$ & AUAAACAACCC \\
87 & $8.30002 \mathrm{e}-06$ & AUAGGGAUUGC \\
379 & $7.18559 \mathrm{e}-06$ & AAAUAAAGCGC \\
698 & $7.0473 \mathrm{e}-06$ & AAACGACGCGG \\
2 & $6.71422 \mathrm{e}-06$ & AACUCGUUUCA \\
377 & $6.18301 \mathrm{e}-06$ & AUAAAGCGCCA \\
378 & $5.15652 \mathrm{e}-06$ & AAUAAAGCGCC \\
342 & $3.84459 \mathrm{e}-06$ & AAUUAUAAACA \\
17 & $2.39015 \mathrm{e}-06$ & AGCAAACAGGG \\
10 & $2.21362 \mathrm{e}-06$ & AGGGUAAUAC \\
356 & $1.05458 \mathrm{e}-06$ & CUUAUCCGUUC \\
\hline
\end{tabular}

Fig. 3. (A) This plot was created according to the all tips for evaluating accessible local structures using the Sfold Web server (sites with high probabilities are predicted to be accessible). Loop probability profiles of the fimH mRNA (nucleotide position 1-400) including (B) Interior plot, (C) Bulge plot, (D) Hairpin plot and (E) Multi-branched plot. (F) Results of RNA Structure 5.5 (ASO Length has been considered $11 \mathrm{bp}$ ). Successful ASO design also needs to take into consideration thermodynamic energy. In this study, the program OligoWalk from the package RNA structure was used for calculating thermodynamic properties between the ASO and mRNA target sequence. To design a potent ASO, the binding energy between the ASO and mRNA should be $\Delta \mathrm{G}_{37} \geq-8 \mathrm{kcal} / \mathrm{mol}$, whereas the energy for binding between ASOs should be $\Delta \mathrm{G}_{37} \geq-1.1 \mathrm{kcal} / \mathrm{mol}$ (28). 
(A)

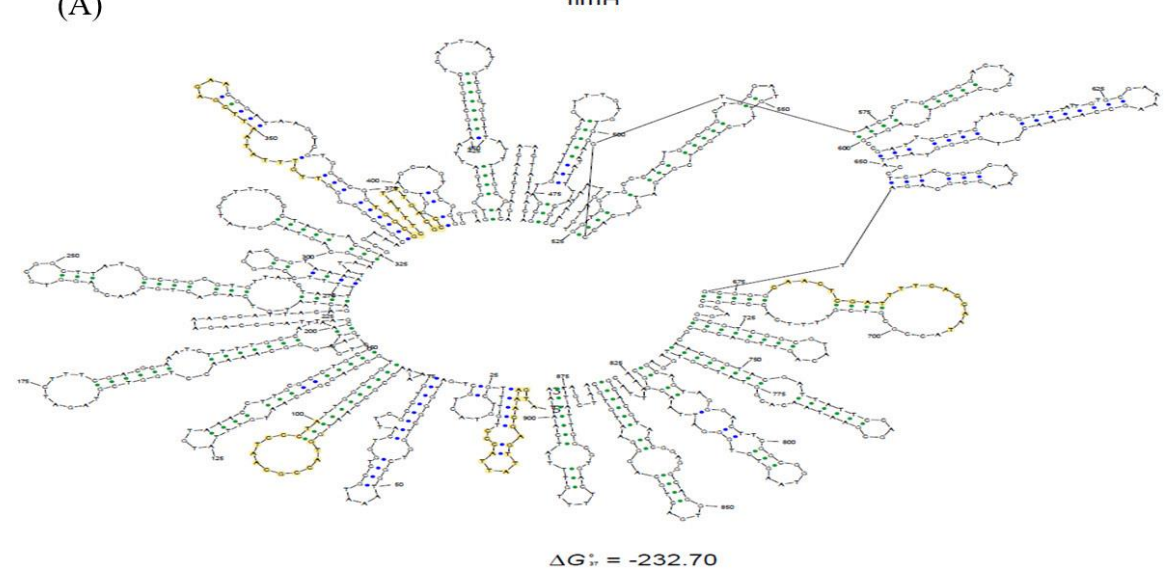

(B)

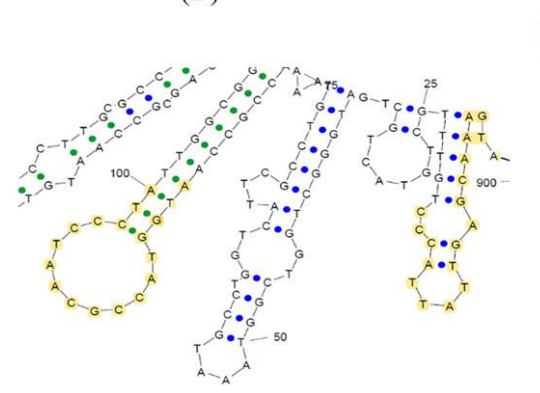

(C)
(D)

Fig. 4. The regions of fimH mRNA targeted in this study were highlighted in yellow. (A) Predicted secondary structure of fimH mRNA of $E$. coli UTI89 strain with Mfold. The target region of (B) antisense oligonucleotides number 1 (nucleotides 1-18) and number 2 (nucleotides 84-101), (C) antisense oligonucleotides number 3 (nucleotides 341-358) and number 4 (nucleotides 377-394) and (D) antisense oligonucleotide number 5 (nucleotides 691-708).

Table 3. Antisense oligonucleotides which targeted the predicted accessible regions of fimH mRNA and binding parameters predicted by RNA structure

\begin{tabular}{lcccccc}
\hline No) Oligo sequence $(\mathbf{5} \rightarrow \mathbf{3})$ & Target site $^{\mathbf{a}}$ & Overall $\Delta \mathbf{G}$ & Oligo-self $\Delta \mathbf{G}$ & Oligo-oligo $\Delta \mathbf{G}$ & $\mathbf{T m}^{\mathbf{c}}$ & $\mathbf{G C}(\boldsymbol{\%})$ \\
\hline 1) GGTAATAACTCGTTTCAT & $1-18$ & -13.4 & 0 & -6.4 & 55 & 53 \\
2) ATAGGGATTGCGGTACCA & $84-101$ & -16 & -1 & -6.3 & 60 & 50 \\
3) TTCTCGAATTATAAACAA & $341-358$ & -11 & 0 & -8 & 50 & 40 \\
4) GCGTCAAATAAAGCGCCA & $377-394$ & -15.5 & 0 & -5.8 & 63 & 50 \\
5) AAACGACGCGGTATTGGT & $691-708$ & -13.8 & 0 & -7.7 & 64 & 50 \\
6) CTAAAAAACTTGGTTAAG & Control sequence & ND & ND & ND & ND & ND \\
\hline
\end{tabular}

ND: not determined

a fimH gene sequence in GenBank with AC number: NC_007946.1

$\Delta \mathrm{G}(\mathrm{kcal} / \mathrm{mol})$ means free energy; index for each parameter: overall $\Delta \mathrm{G} \leq-10 \mathrm{kcal} / \mathrm{mol}$, oligo-self $\Delta \mathrm{G} \geq$

$-2 \mathrm{kcal} / \mathrm{mol}$, oligo-oligo $\Delta \mathrm{G} \geq-10 \mathrm{kcal} / \mathrm{mol}, \mathrm{GC} \%=40-60 \%$ and $\mathrm{Tm}>50^{\circ} \mathrm{C}$ (28).

ASOs-based antibacterial agent have been represented as an effective approach in suppressing bacterial gene expression and cell growth, making this strategy promising for developing highly specific antibacterial agents (36). In the last decades, on top of targeting the essential genes, non-essential genes required for virulence, such as those that confer invasiveness or biofilm formation, were also targeted 


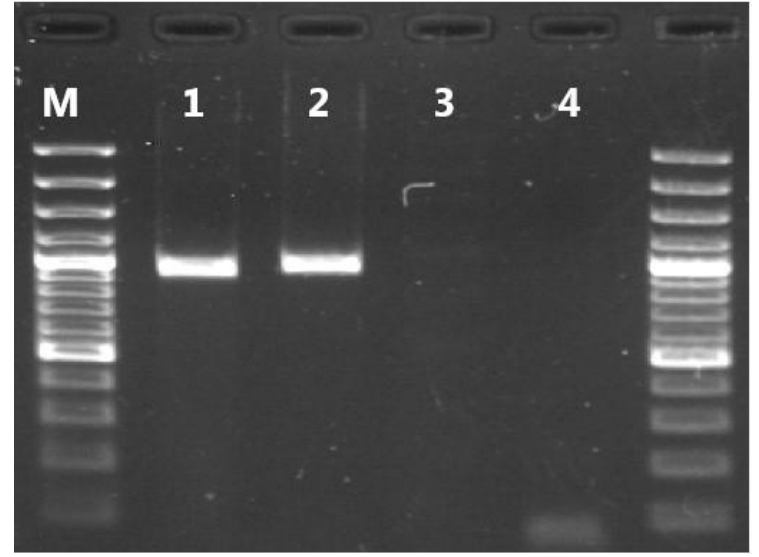

Fig. 5. Agarose gel electrophoresis showing:

1, PCR amplification of fimH gene with primers without T7 promoter (903 bp)

2, PCR amplification of fimH gene with primers with T7 promoter (933 bp)

3 , none template control (NTC)

4, Control negative

M, 100 bp ladder

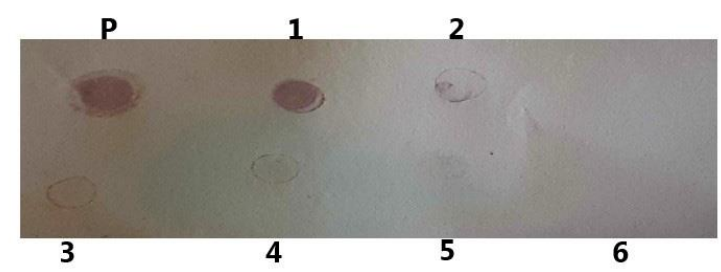

Fig. 6. Dot-blot hybridization analysis of the binding activity of predicted oligonucleotides number 1 to 6 ( $3 \mu \mathrm{g}$ fixed on membrane) with $100 \mathrm{ng} / \mathrm{mL}$ of $E$. coli DIG labeled fimH mRNA in DIG Easy Hybridization solution. Antisense oligonucleotide number 1 which targeted the region contains start codon of fim $H$ mRNA showed the strongest hybridization signal, whereas the negative control (oligonucleotide number 6) showed no hybridized signal. P: Positive identification control (DIG -labeled antisense RNA fixed on membrane).

by antisense oligomers. Reducing the resistance risk could be a merit of targeting a non-essential gene (37). Hu et al. (19) targeted the mRNA of the motA gene (target region 262-273), encoding the element of the flagella motor complex, in Pseudomonas aeruginosa. In their study, antisense peptide nucleic acids (PNA) conjugated the peptide, targeting the mRNA of the motA could inhibit biofilm formation in $P$. aeruginosa $\mathrm{PAO} 1$ strain in a dose-dependent manner. Da et al. (25) also targeted agrA mRNA (target re- gion 17-34) with peptide-conjugated locked nucleic acids (PLNAs) to inhibit $a g r$ activity and arrest the pathogenicity of community-associated methicillin-resistant Staphylococcus aureus (CA-MRSA).

One of the major challenges in antisense antimicrobials approaches is identification of effective target sites. A review of the literature on antibacterial ASOs shows that the site of translation initiation and the start codon region are seen as the ideal mRNA target region for antisense suppression $(22,38,39)$. Start codon region is accessible for ribosome assembly and also targeted with natural examples of antisense (40). However, some researcher pointed out that the full length of a target gene screening using computational prediction and dot-blot hybridization could help us to select potent and specific ASOs compounds (19, 23, 24). They first predicted secondary structure of target genes mRNA through computational analysis and then checked the accessibility of target mRNA by performing dot-blot hybridization. In contrary to their studies, our investigation demonstrated that the start codon region of $f i m H$ as the suitable mRNA target site for antisense suppression.

In this study, we first appraised the conserved regions of fimH gene in the whole genome sequenced strains of E. coli. Then, based on the predicted secondary structure and thermodynamic properties, accessible regions in mRNA of fimH were determined and oligonucleotides complementary to these regions were synthesized and binding activity of these oligonucleotides to the fimH DIG labeled mRNA was assessed with dot-blot hybridization. Dot-blot hybridization results indicated the antisense oligonucleotide number one that targeted the region contains start codon of fimH mRNA showed the strongest hybridization signal, whereas the negative control showed no hybridized signal. The start codon region of fim $\mathrm{H}$ mRNA was also predicted by the RNA structure program as a site with highest probability to be accessible with antisense oligonucleotide. Considering that we concluded the region contains start codon of fimH mRNA as the ideal mRNA target site for antisense suppression compares to the other sites.

Our bioinformatics analysis verified that the fimH coding gene in most of $E$. coli strains such as K12, UTI89, W3110 and LF82 was 903 bp in length, while the length was 912 bp for both E. coli CFT073 (UPEC) and E. coli 536 (UPEC) strains. The difference was related to the nine bases in the first part of the gene. Searching FimH protein in UniProt da- 
tabase was also confirmed this variation in protein length (Fig. 2B). Therefore, it is recommended in the future both of these sites (Fig. 2) be targeted with anti-fimH antisense compounds and the effectiveness and specificity of RNA silencing be verified by western blotting analysis. Moreover, the capacity of anti-fim $H$ antisense compounds to inhibit the adherence of UPEC strains to epithelial bladder cells (anti-adhesive activity) could be assessed by cell culture methodologies.

In present study, a structure-based target site selecting method, with an emphasis on its ultimate effectiveness was presented. We found the conserved structures among numerous optimal and suboptimal mRNA predicted secondary structures. Then, we identified the appropriate RNA local structures, sites with high probability to be accessible with antisense oligonucleotide, including the internal loops (targeted with antisense oligonucleotide number 3), external loops (targeted with antisense oligonucleotides number 2 and 5), joint sequences(targeted with antisense oligonucleotide number 4 ), hairpins and bulges of 10 or more sequential nucleotides. Finally, binding energy $(\Delta \mathrm{G} 37)$ was predicted by RNA structure software.

Considering the critical role of FimH adhesion for initiating and establishment of UPEC infections, this virulence factor could be an attractive target for the development of anti-virulence antisense therapeutics. The results obtained here demonstrate that the region contains start codon of fim $\mathrm{H}$ mRNA could act as the potential mRNA target site for anti-fimH antisense therapeutics.

\section{ACKNOWLEDGEMENTS}

This project honorably sponsored financially by the Pasteur Institute of Iran.

\section{REFERENCES}

1. Barber AE, Norton JP, Wiles TJ, Mulvey MA. Strengths and limitations of model systems for the study of urinary tract infections and related pathologies. Microbiol Mol Biol Rev 2016;80:351-367.

2. Tabasi M, Asadi Karam MR, Habibi M, Yekaninejad MS, Bouzari S. Phenotypic assays to determine virulence factors of uropathogenic Escherichia coli (UPEC) isolates and their correlation with antibiotic resistance pattern. Osong Public Health Res Perspect 2015;6:261-268

3. Flores-Mireles AL, Walker JN, Caparon M, Hultgren SJ. Urinary tract infections: epidemiology, mechanisms of infection and treatment options. Nat Rev Microbiol 2015;13:269-284.

4. Wilson ML, Gaido L. Laboratory diagnosis of urinary tract infections in adult patients. Clin Infect Dis 2004;38:1150-1158.

5. Loubet P, Ranfaing J, Dinh A, Dunyach-Remy C, Bernard $\mathrm{L}$, Bruyère $\mathrm{F}$, et al. Alternative therapeutic options to antibiotics for the treatment of urinary tract infections. Front Microbiol 2020;11:1509.

6. Saint S, Kowalski CP, Kaufman SR, Hofer TP, Kauffman CA, Olmsted RN, et al. Preventing hospital-acquired urinary tract infection in the United States: a national study. Clin Infect Dis 2008;46:243-250.

7. Asadi Karam MR, Habibi M, Bouzari S. Urinary tract infection: pathogenicity, antibiotic resistance and development of effective vaccines against Uropathogenic Escherichia coli. Mol Immunol 2019;108:56-67.

8. Schwartz DJ, Kalas V, Pinkner JS, Chen SL, Spaulding CN, Dodson KW, et al. Positively selected FimH residues enhance virulence during urinary tract infection by altering FimH conformation. Proc Natl Acad Sci U S A 2013;110:15530-15537.

9. Habibi M, Asadi Karam MR, Bouzari S. Transurethral instillation with fusion protein $\mathrm{MrpH}$. FimH induces protective innate immune responses against uropathogenic Escherichia coli and Proteus mirabilis. APMIS 2016;124:444-452.

10. Bien J, Sokolova O, Bozko P. Role of uropathogenic Escherichia coli virulence factors in development of urinary tract infection and kidney damage. Int $J$ Nephrol 2012;2012:681473.

11. Cozens D, Read RC. Anti-adhesion methods as novel therapeutics for bacterial infections. Expert Rev Anti Infect Ther 2012;10:1457-1468.

12. Hung CS, Dodson KW, Hultgren SJ. A murine model of urinary tract infection. Nat Protoc 2009;4:1230-1243.

13. Vogel J. An RNA biology perspective on species-specific programmable RNA antibiotics. Mol Microbiol 2020;113:550-559.

14. Skvortsova YV, Salina EG, Burakova EA, Bychenko OS, Stetsenko DA, Azhikina TL. A new antisense phosphoryl guanidine oligo-2'-O-methylribonucleotide penetrates into intracellular mycobacteria and suppresses target gene expression. Front Pharmacol 2019;10:1049.

15. Sully EK, Geller BL. Antisense antimicrobial therapeutics. Curr Opin Microbiol 2016;33:47-55.

16. Oh E, Zhang Q, Jeon B. Target optimization for peptide nucleic acid (PNA)-mediated antisense inhibition of the CmeABC multidrug efflux pump in Campylobacter 
jejuni. J Antimicrob Chemother 2014;69:375-380.

17. Sturge CR, Felder-Scott CF, Pifer R, Pybus C, Jain $\mathrm{R}$, Geller BL, et al. AcrAB-TolC inhibition by peptide-conjugated phosphorodiamidate Morpholino oligomers restores antibiotic activity in vitro and in vivo. ACS Infect Dis 2019;5:1446-1455.

18. Alajlouni RA, Seleem MN. Targeting Listeria monocytogenes rpoA and rpoD genes using peptide nucleic acids. Nucleic Acid Ther 2013;23:363-367.

19. Xia Y, Xiong Y, Li X, Su X. Inhibition of biofilm formation by the antisense peptide nucleic acids targeted at the motA gene in Pseudomonas aeruginosa PAO1 strain. World J Microbiol Biotechnol 2011;27:19811987.

20. Jackson A, Jani S, Sala CD, Soler-Bistué AJ, Zorreguieta A, Tolmasky ME. Assessment of configurations and chemistries of bridged nucleic acids-containing oligomers as external guide sequences: a methodology for inhibition of expression of antibiotic resistance genes. Biol Methods Protoc 2016;1(1):bpw001.

21. Nejad AJ, Shahrokhi N, Nielsen PE. Targeting of the essential acpP, ftsZ, and rne genes in carbapenem-resistant Acinetobacter baumannii by antisense PNA precision antibacterials. Biomedicines 2021;9:429.

22.Good L, Stach JE. Synthetic RNA silencing in bacteriaantimicrobial discovery and resistance breaking. Front Microbiol 2011;2:185.

23. Liang S, He Y, Xia Y, Wang H, Wang L, Gao R, et al. Inhibiting the growth of methicillin-resistant Staphylococcus aureus in vitro with antisense peptide nucleic acid conjugates targeting the ftsZ gene. Int J Infect Dis 2015;30:1-6.

24. Wang H, He Y, Xia Y, Wang L, Liang S. Inhibition of gene expression and growth of multidrug-resistant Acinetobacter baumannii by antisense peptide nucleic acids. Mol Biol Rep 2014;41:7535-7541.

25. Da F, Yao L, Su Z, Hou Z, Li Z, Xue X, et al. Antisense locked nucleic acids targeting agrA inhibit quorum sensing and pathogenesis of community-associated methicillin-resistant Staphylococcus aureus. J Appl Microbiol 2017;122:257-267.

26. Kent WJ. BLAT--the BLAST-like alignment tool. Genome Res 2002;12:656-664.

27. Zuker M. Mfold web server for nucleic acid folding and hybridization prediction. Nucleic Acids Res 2003;31:3406-3415.

28. Mathews DH, Turner DH. Prediction of RNA second- ary structure by free energy minimization. Curr Opin Struct Biol 2006;16:270-278.

29. Ding Y, Chan CY, Lawrence CE. Sfold web server for statistical folding and rational design of nucleic acids. Nucleic Acids Res 2004;32(Web Server issue):W135-W141.

30. Reuter JS, Mathews DH. RNAstructure: software for RNA secondary structure prediction and analysis. BMC Bioinformatics 2010;11:129.

31. Eisel D, Seth O, Grünewald-Janho S, Kruchen B, Rüger B (2008). DIG application manual for filter hybridization. Roche Diagnostics GmbH. Mannheim. Germany.

32. Hart SM, Basu C. Optimization of a digoxigenin-based immunoassay system for gene detection in Arabidopsis thaliana. J Biomol Tech 2009;20:96-100.

33. Cusumano CK, Pinkner JS, Han Z, Greene SE, Ford BA, Crowley JR, et al. Treatment and prevention of urinary tract infection with orally active FimH inhibitors. Sci Transl Med 2011;3:109ra-ra115.

34. Jarvis C, Han Z, Kalas V, Klein R, Pinkner JS, Ford B, et al. Antivirulence isoquinolone mannosides: optimization of the biaryl aglycone for FimH lectin binding affinity and efficacy in the treatment of chronic UTI. ChemMedChem 2016;11:367-373.

35. Schembri MA, Kjaergaard K, Sokurenko EV, Klemm P. Molecular characterization of the Escherichia coli FimH adhesin. J Infect Dis 2001;183 Suppl 1:S28-S31.

36. Bai H, You Y, Yan H, Meng J, Xue X, Hou Z, et al. Antisense inhibition of gene expression and growth in gram-negative bacteria by cell-penetrating peptide conjugates of peptide nucleic acids targeted to rpoD gene. Biomaterials 2012;33:659-667.

37. Wojciechowska M, Równicki M, Mieczkowski A, Miszkiewicz J, Trylska J. Antibacterial peptide nucleic acids - facts and perspectives. Molecules 2020;25:559.

38. Dryselius R, Aswasti SK, Rajarao GK, Nielsen PE, Good L. The translation start codon region is sensitive to antisense PNA inhibition in Escherichia coli. Oligonucleotides 2003;13:427-433.

39. Daly SM, Sturge CR, Marshall-Batty KR, Felder-Scott $\mathrm{CF}$, Jain R, Geller BL, et al. Antisense inhibitors retain activity in pulmonary models of Burkholderia infection. ACS Infect Dis 2018;4:806-814.

40. Rasmussen LC, Sperling-Petersen HU, Mortensen KK. Hitting bacteria at the heart of the central dogma: sequence-specific inhibition. Microb Cell Fact 2007;6:24. 